"C 2019 IEEE. Personal use of this material is permitted. Permission from IEEE must be obtained for all other uses, in any current or future media, including reprinting/republishing this material for advertising or promotional purposes, creating new collective works, for resale or redistribution to servers or lists, or reuse of any copyrighted component of this work in other works." 


\title{
Wide-Angle Wideband Frequency-Independent Beam-Scanning Leaky Wave Antenna
}

\author{
Shu-Lin Chen, Debabrata K. Karmokar, Richard W. Ziolkowski, and Y. Jay Guo \\ Global Big Data Technologies Centre (GBDTC) \\ University of Technology Sydney (UTS) \\ Ultimo, Australia \\ Shulin.Chen@uts.edu.au
}

\begin{abstract}
Frequency-independent beam scanning leaky-wave antennas (LWAs) that can operate over a specific frequency band are highly desired for the future wireless systems. A composite right/left-handed (CRLH) LWA is developed in this paper that facilitates this functionality. It utilizes two groups of varactor diodes to realize the frequency-independent beam scanning capability. The final reconfigurable CRLH LWA with simple DC biasing network can achieve an over $100^{\circ}$ frequencyindependent beam scan at each frequency point between 4.75 and $5.25 \mathrm{GHz}$ in the simulations. An antenna prototype was fabricated and tested. The measured results at $5 \mathrm{GHz}$ confirm its simulated performance characteristics.
\end{abstract}

Index Terms-Beam scanning, composite right/left-handed (CRLH), frequency-independent, leaky-wave antenna (LWA)

\section{INTRODUCTION}

Leaky-wave antennas (LWAs) are highly desired for the future wireless systems that require low-cost, easy-integration and multiple functionalities. Generally, they serially leak power from open regions of a guiding wave structure, and form focused beams in the far field regions with inherent beam scanning ability [1].

One issue of the LWAs has been an open stopband. It occurs as reflections accumulate at the input when the antenna's main beam points to broadside direction, and hence the broadside radiation is significantly suppressed [2]. Composite right/lefthanded (CRLH) structure is a popular technique that can eliminate the open stopband. It introduces left-handed characteristics into traditional right-handed transmission lines, and a continuous transition between $\mathrm{LH}$ and $\mathrm{RH}$ curves is achieved when a balanced condition is obtained [3].

Although successful advancements have been achieved on CRLH LWAs, most of them are frequency-based, i.e., the main beam scans with source frequency. Whilst, it prefers to realize frequency-independent beam scanning capability at a specific allocated band for most wireless communication systems. To date, only a few interesting solutions on the CRLH LWAs have been reported to target this issue. It was reported in [4] that a varactor-loaded CRLH microstrip LWA can realize a frequency-independent beam scanning from backward $\left(-10^{\circ}\right)$ to forward $\left(+7.5^{\circ}\right)$ direction through broadside. The frequencyindependent beam scanning range was enlarged to $66^{\circ}$ in [5] by tuning two groups of capacitor values. However, this configuration is hard to incorporate practical DC biasing lines. Liquid-crystal CRLH LWA was developed in [6] that can achieve a frequency-independent beam scanning from $-21^{\circ}$ to $+23^{\circ}$ at $12.4 \mathrm{GHz}$. Note that the above CRLH structures can only realize the frequency-independent beam scanning capability at a single frequency rather than a desired wide frequency band. Other structures have been also reported to scan the main beam with a frequency-dependant behaviour. A modulated corrugated microstrip line was employed in [7] to achieve $45^{\circ}$ frequency-dependant beam scan between 5.5 and $5.8 \mathrm{GHz}$. A microstrip line LWA with a reconfigurable 2bit phase unit was reported in [8] that radiates five switchable beams at $5 \mathrm{GHz}$, and five prototypes were fabricated to validate these five working states. It is noted that these reported designs usually have a limited scanning range, and the frequencyindependent operating band is narrow.

In this paper, a CRLH LWA with a simple configuration is developed that can realize a frequency-independent continuous beam scanning over a wide bandwidth. Two groups of varactor diodes are incorporated to control the dispersion behaviors electronically, and hence to realize a frequency-independent beam scan. Simple yet effective DC networking has been introduced for the reconfiguration, and the final frequencyindependent CRLH LWA is attained. Simulations indicate that the antenna can operate from 4.75 to $5.25 \mathrm{GHz}$ and frequencyindependent beam scanning in a large angle, i.e., $\geq 100^{\circ}$, has been achieved for each frequency point. An antenna prototype is fabricated and assembled. Measured results at $5 \mathrm{GHz}$ have validated the antenna's frequency-independent scanning in a wide angle range.

\section{Antenna Configuration And Analysis}

\section{A. Antenna Configuration}

The developed wide-angle wideband frequency-independent beam-scanning leaky wave antenna consists of the upper substrate, lower substrate and three copper layers, i.e., patch layer, ground layer and biasing layer. Both substrates are F4BM-2 produced by Wangling Company with the permittivity $\varepsilon_{r}=$ 2.2 and loss tangent $\tan \delta=0.001$. The height of the upper and lower substrates is 1.575 and $0.8 \mathrm{~mm}$, respectively.

The patch layer was printed on top of the upper substrate and the ground layer was printed on the bottom face. Configurations of the patch layer and ground layer are shown in Fig. 1 (a) and (b), respectively. Main patches etched with meander gaps were printed on the patch layer. The dimensions of the 


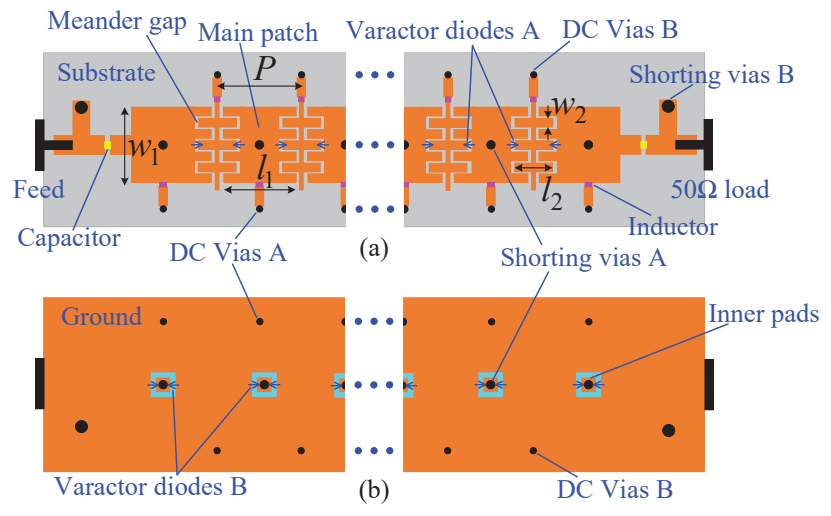

Fig. 1. The upper substrate of the fixed-frequency beam scanning CRLH LWA. (a) Patch layer. (b) Ground layer.

main patches are represented by $l_{1}, l_{2}, w_{1}, w_{2}$, and $P$. Two groups of small pads were printed and connected to DC vias $\mathrm{A}$ and $\mathrm{B}$. There are inductors between the small pads and the main patches used for RF choke. A group of varactor diodes A was soldered between the main patches and the meander gaps. As seen in Fig. 1 (b), small gaps were etched between the inner pads and the ground plane, and a group of varactor diodes B was placed across these small gaps. Shorting vias A were centrally located to connect the main patches to the inner pads. The diameter of the shorting via $\mathrm{A}$ is $d_{A}$.

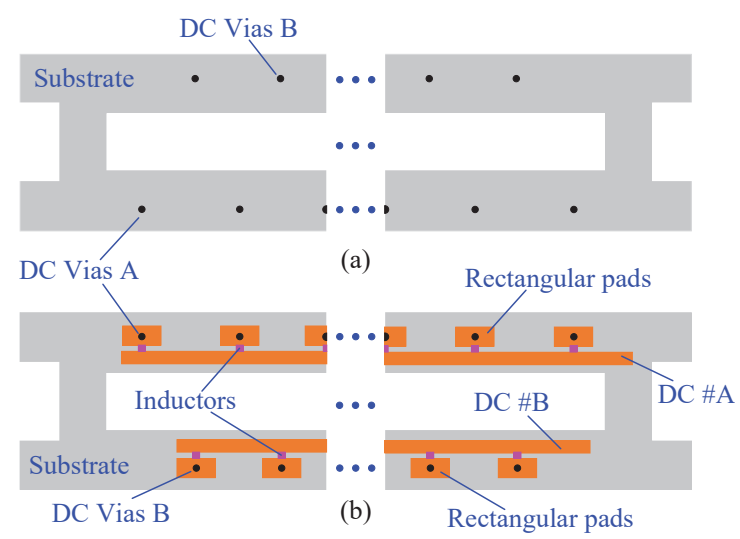

Fig. 2. The lower substrate of the fixed-frequency beam scanning CRLH LWA. (a) Top layer of the lower substrate. (b) Biasing layer.

Fig. 2 (a) and (b) show the top and bottom layers of the lower substrate, respectively. All of the metal on the top was removed, and the bottom face is the biasing layer. Two long DC biasing lines DC \#A and \#B along with two groups of rectangular pads were printed on the biasing layer. These rectangular pads are connected to $\mathrm{DC}$ vias $\mathrm{A}$ and $\mathrm{B}$. Inductors were placed between the rectangular pads and the long DC biasing lines for RF choke. In this manner, it forms a simple yet effective DC biasing network. All of the positive polarities of varactor diodes $\mathrm{A}$ are connected, and each connects to DC \#A through a small pad, a DC via A and a rectangular pad. Similarly, all of the negative polarities of varactor diodes
TABLE I

OPtiMized DESIGN PARAMETER VALUES (IN MILliMETERS) OF THE DEVELOPED FREQUENCY-INDEPENDENT CRLH LWA

\begin{tabular}{cccccccc}
\hline \hline Parameters & $l_{1}$ & $l_{2}$ & $w_{1}$ & $w_{2}$ & $P$ & $d_{A}$ & $d_{B}$ \\
\hline Values $(\mathrm{mm})$ & 13 & 4 & 14 & 1 & 14 & 1 & 1.5 \\
\hline \hline
\end{tabular}

A are connected to DC \#B. All of the positive polarities of varactor diodes $\mathrm{B}$ are connected to the ground plane, and the negative polarities are connected to the inner pads, and hence, the DC \#A. Consequently, a DC power source can be applied between DC \#A and DC \#B to bias all of the varactor diodes A simultaneously. All of the varactor diodes B can be biased when the source is applied between DC \#A and the ground plane. Note that a continuous rectangular slot was cut between the DC \#A and DC \#B on the lower substrate due to the significant bumps caused by the soldering. Similar rectangular cuts were made at both ends to place the feed and $50 \Omega$ load.

This antenna has nine unit cells. It feeds from the left and terminates with $50 \Omega$ load on the right. The shorting vias B connected with the stubs on each end are used for impedance matching. The diameter of the shorting via $\mathrm{B}$ is $d_{B}$. Capacitors are placed on the gaps between the feed line and the main patches for blocking DC signals. The optimized values for the antenna parameters are given in Table I.

\section{B. Operating mechanism}

This developed frequency-independent LWA can be equivalent to a typical T-type CRLH circuit [3], where $L_{R}$ and $C_{R}$ denote the right-handed inductance and capacitance, respectively, and $L_{L}$ and $C_{L}$ are left-handed inductance and capacitance. The $L_{R}$ and $C_{R}$ are naturally associated with the microstrip transmission line properties. The $L_{L}$ is introduced by the shorting vias $\mathrm{A}$ that connect the main patches to the inner pads. The meander gaps between adjacent main patches introduce the $C_{L}$. Note that varactor diodes $\mathrm{A}$ are located between these meander gaps, and hence can reconfigure the $C_{L}$. Varactor diodes B are placed between the inner pads and the ground plane to control the $L_{L}$. Therefore, both of the $L_{L}$ and $C_{L}$ can be reconfigured individually, and hence, to tune the dispersion properties of the CRLH LWA. Frequencyindependent beam-scanning can be obtained from the variable dispersion performance [5].

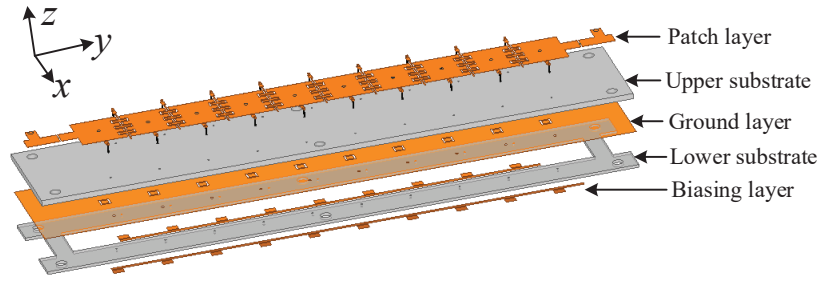

Fig. 3. Explosive view of the developed frequency-independent CRLH LWA. 
TABLE II

Simulated Performance of the Frequency-Independent Beam SCANning CRLH LWA Operating at 4.75, 5, and 5.25 GHz

\begin{tabular}{|c|c|c|c|c|c|c|c|c|c|c|c|c|c|c|c|c|c|c|}
\hline \multirow{3}{*}{$\begin{array}{c}\text { Antenna performance } \\
\text { State }\end{array}$} & \multicolumn{18}{|c|}{ Operating frequency } \\
\hline & \multicolumn{6}{|c|}{$4.75 \mathrm{GHz}$} & \multicolumn{6}{|c|}{$5 \mathrm{GHz}$} & \multicolumn{6}{|c|}{$5.25 \mathrm{GHz}$} \\
\hline & 1 & 2 & 3 & 4 & 5 & 6 & 1 & 2 & 3 & 4 & 5 & 6 & 1 & 2 & 3 & 4 & 5 & 6 \\
\hline$C_{V 1}(\mathrm{pF})$ & 0.16 & 0.19 & 0.25 & 0.35 & 0.6 & 0.7 & 0.1 & 0.12 & 0.18 & 0.25 & 0.45 & 0.7 & 0.08 & 0.12 & 0.16 & 0.18 & 0.35 & 0.5 \\
\hline$C_{V 2}(\mathrm{pF})$ & 0.85 & 0.86 & 1.05 & 1.2 & 2 & 3.5 & 0.75 & 0.82 & 0.86 & 0.95 & 1.8 & 4 & 0.64 & 0.68 & 0.71 & 0.86 & 1.7 & 4 \\
\hline$S_{11}(\mathrm{~dB})$ & -7.7 & -9.2 & -13.1 & -10.9 & -11.1 & -10.5 & -9.0 & -21.2 & -17.8 & -11.0 & -9.3 & -10.2 & -9.0 & -15.5 & -13.2 & -10.0 & -7.6 & -9.3 \\
\hline$S_{21}(\mathrm{~dB})$ & -40.7 & -38.3 & -11.5 & -6.7 & -4.2 & -4.3 & -43.7 & -26.8 & -15.1 & -8.8 & -6.1 & -3.9 & -46.9 & -34.4 & -22.7 & -10.8 & -7.8 & -5.9 \\
\hline Main beam angle & $-46^{\circ}$ & $-22^{\circ}$ & $0^{\circ}$ & $+18^{\circ}$ & $+36^{\circ}$ & $+54^{\circ}$ & $-46^{\circ}$ & $-24^{\circ}$ & $0^{\circ}$ & $+14^{\circ}$ & $+34^{\circ}$ & $+62^{\circ}$ & $-42^{\circ}$ & $-20^{\circ}$ & $0^{\circ}$ & $+20^{\circ}$ & $+36^{\circ}$ & $+58^{\circ}$ \\
\hline Realized gain (dBi) & 7.5 & 7.5 & 8.1 & 8.7 & 8.0 & 8.4 & 7.2 & 6.9 & 8.3 & 9.7 & 9.3 & 7.2 & 6.1 & 6.7 & 7.1 & 10.3 & 9.6 & 10.1 \\
\hline
\end{tabular}

\section{Simulated Results}

A model of this developed frequency-independent CRLH LWA was set up and simulated, and its explosive view is shown in Fig. 3. The simulation results indicated that this frequency-independent beam scanning LWA would operate over a $10 \%$ frequency bandwidth, i.e., 4.75 to $5.25 \mathrm{GHz}$, and for each frequency point within the bandwidth, the main beam can be continuously scanned from the backward to forward directions through broadside. Table II summarizes the simulated performance of the optimized LWA working at $4.75,5$, and $5.25 \mathrm{GHz}$. It is noted that the varactor diodes A and $\mathrm{B}$ were treated as lossless for these simulation studies, i.e., they were simply equivalent to variable capacitors with capacitances $C_{V 1}$ and $C_{V 2}$, respectively.

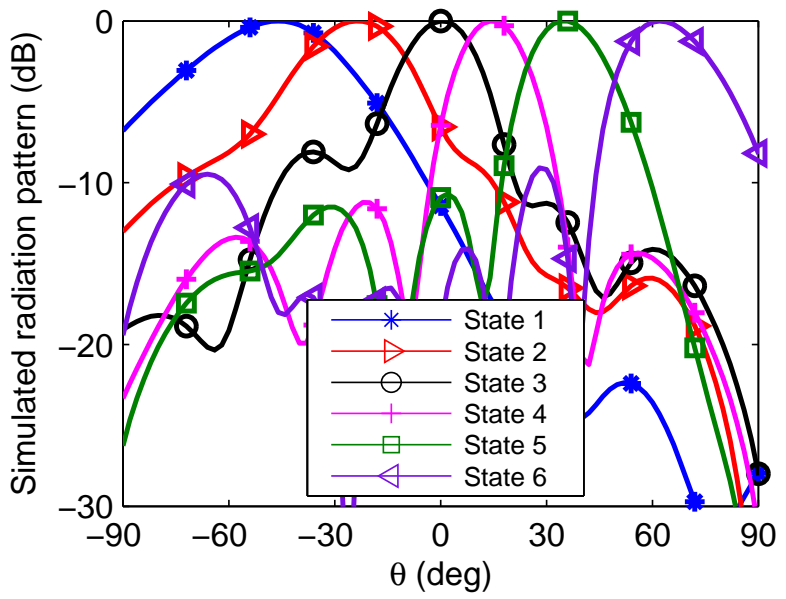

Fig. 4. Simulated radiation patterns for antenna's six operating states at 5 GHz.

At $5 \mathrm{GHz}$, the beam scanning range is $108^{\circ}$, from $-46^{\circ}$ to $+62^{\circ}$, when the values of $\left(C_{V 1}, C_{V 2}\right)$ increase from $(0.1 \mathrm{pF}$, $0.75 \mathrm{pF})$ to $(0.7 \mathrm{pF}, 4 \mathrm{pF})$. The simulated patterns for antenna's six operating states are shown in Fig. 4. It is noted that the half-power beamwidths (HPBWs) of the backward beams are wider than those of the forward beams. This occurs because
TABLE III

MEASURed Performance CHARACTERISTICS OF THE FREQUENCY-INDEPENDENT BEAM SCANNING CRLH LWA OPERATING AT $5 \mathrm{GHz}$

\begin{tabular}{c|ccccc}
\hline \hline \multirow{2}{*}{ Antenna performance } & \multicolumn{5}{|c}{ State } \\
\cline { 2 - 6 } & 1 & 2 & 3 & 4 & 5 \\
\hline Voltage $V_{1}(\mathrm{~V})$ & 18 & 18 & 10.5 & 3.1 & 2.4 \\
\hline Voltage $V_{2}(\mathrm{~V})$ & 16 & 13 & 11 & 2 & 0.1 \\
\hline$S_{11}(\mathrm{~dB})$ & -16.8 & -12.9 & -10.7 & -9.6 & -10.0 \\
\hline$S_{21}(\mathrm{~dB})$ & -39.9 & -34.7 & -35.3 & -18.3 & -20.7 \\
\hline Main beam angle & $-37^{\circ}$ & $-19^{\circ}$ & $0^{\circ}$ & $+24^{\circ}$ & $+32^{\circ}$ \\
\hline Realized gain $(\mathrm{dBi})$ & 5.7 & 4.9 & 5.4 & 5.7 & 4.6 \\
\hline \hline
\end{tabular}

the radiation in the first few states (backward beam angles) is generated primarily by only the first few unit cells of the LWA while most of them contribute to it for the latter few states (forward beam angles). The sidelobe levels are lower than $-8.1 \mathrm{~dB}$ for all these six patterns. The realized gain variation is now $2.8 \mathrm{~dB}$, i.e., from $6.9-9.7 \mathrm{dBi}$. When $\left(C_{V 1}\right.$, $\left.C_{V 2}\right)=(0.18 \mathrm{pF}, 0.86 \mathrm{pF})$, the main beam points into the broadside direction and the peak realized gain is $8.3 \mathrm{dBi}$. The values of $\left|S_{11}\right|$ remain below $-9.0 \mathrm{~dB}$ for all the six operating states. The values of $\left|S_{21}\right|$ increase from $-43.7 \mathrm{~dB}$ to -3.9 $\mathrm{dB}$ when $\left(C_{V 1}, C_{V 2}\right)$ is varied within the noted range.

\section{Measured Results}

A prototype of the developed frequency-independent CRLH LWA was fabricated to verify the above simulated performance. Two types of commercial varactors were used to realize the capacitance tuning, i.e., MA46H120 is used for the varactor diodes A and SMV1405 is used for varactor diodes B. The far-field radiation characteristics of the antenna were measured using a spherical near-field (SNF) antenna measurement system NSI-700S-50 located at CSIRO, Australia.

Measurements were conducted at $5 \mathrm{GHz}$ for an illustration. Table III presents the measured performance of the frequencyindependent beam scanning CRLH LWA for five working 
states at $5 \mathrm{GHz}$. It is seen that the measured beam scanning range is from $-37^{\circ}$ to $+32^{\circ}$ when $\left(V_{1}, V_{2}\right)$ is varied from $(18 \mathrm{~V}, 16 \mathrm{~V})$ to $(2.4 \mathrm{~V}, 0.1 \mathrm{~V})$. At State 3 , when $\left(V_{1}, V_{2}\right)=$ $(10.5 \mathrm{~V}, 11 \mathrm{~V})$, the main beam points towards the broadside direction with the realized gain $5.4 \mathrm{dBi}$. All the measured values of $\left|S_{11}\right|$ are below $-9.6 \mathrm{~dB}$, and $\left|S_{21}\right|$ are below -18.3 $\mathrm{dB}$. The realized gains are in the range of 4.6 to $5.7 \mathrm{dBi}$ for all the five working states. Note that the measured realized gains are lower than the simulated ones due to the losses on the resistors of the commercial varactors. The measured patterns at those five working states are depicted in Fig. 5. The measured beam scanning range is $69^{\circ}$ at $5 \mathrm{GHz}$ that is reduced from the simulated one. This is caused by the limitations of the capacitance range provided by the commercial varactors.

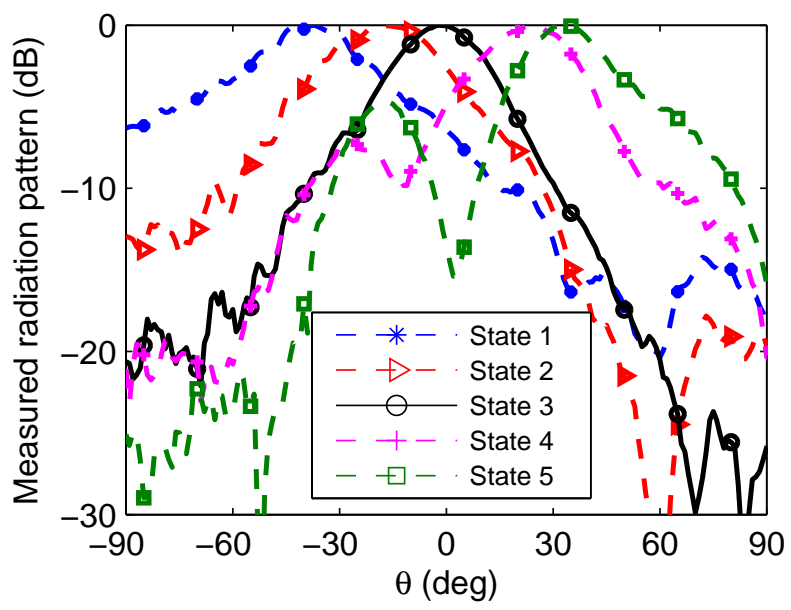

Fig. 5. Measured radiation patterns for antenna's five operating states at 5 GHz.

\section{CONCLUSION}

A frequency-independent continuous beam scan operating over a wide frequency band was achieved by utilizing a simple CRLH LWA with practical DC biasing lines. Two groups of varactor diodes were incorporated to control the left-handed capacitance and inductance, and hence realize the frequencyindependent beam scanning. Simulations indicated that it can continuously scan the main beam with over $100^{\circ}$ angular range in a wide frequency band, i.e., from 4.75 to $5.25 \mathrm{GHz}$. Measurement was conducted at $5 \mathrm{GHz}$ and it confirms that the antenna can continuously scan the main beam from $-37^{\circ}$ to $+32^{\circ}$. The reduced scanning range between the simulation and the measurement is caused by the limitations of the capacitance range provided by the commercial varactors. This realized LWA prototype is a promising candidate for future wireless communication systems.

\section{REFERENCES}

[1] D. R. Jackson, C. Caloz, and T. Itoh, "Leaky-wave antennas," Proc. IEEE, vol. 100, no. 7, pp. 2194-2206, July 2012.

[2] D. K. Karmokar, Y. J. Guo, P. Y. Qin, S. L. Chen, and T. S. Bird, "Substrate integrated waveguide-based periodic backward-to-forward scanning leaky-wave antenna with low cross-polarization," IEEE Trans. Antennas Propag., vol. 66, no. 8, pp. 3846-3856, August 2018.
[3] A. Lai, T. Itoh, and C. Caloz, "Composite right/left-handed transmission line metamaterials," IEEE Microwave Mag., vol. 5, no. 3, pp. 34-50, September 2004.

[4] S. Lim, C. Caloz, and T. Itoh, "Electronically scanned composite right/left handed microstrip leaky-wave antenna," EEE Microw. Wireless Compon. Lett., vol. 14, no. 6, pp. 277-279, June 2004.

[5] A. Suntives and S. V. Hum, "A fixed-frequency beam-steerable halfmode substrate integrated waveguide leaky-wave antenna," IEEE Trans. Antennas Propag., vol. 60, no. 5, pp. 2540-2544, May 2012.

[6] B. Che, T. Jin, D. Erni, F. Meng, Y. Lyu, and Q. Wu, "Electrically controllable composite right/left-handed leaky-wave antenna using liquid crystals in PCB technology," IEEE Trans. Compon. Packag. Manuf. Technol., vol. 7, no. 8, pp. 1331-1342, August 2017.

[7] M. Wang, H. F. Ma, H. C. Zhang, W. X. Tang, X. R. Zhang, and T. J. Cui, "Frequency-fixed beam-scanning leaky-wave antenna using electronically controllable corrugated microstrip line," IEEE Trans. Antennas Propag., vol. 66, no. 9, pp. 4449-4457, September 2018.

[8] L. Chang, Y. Li, Z. Zhang, and Z. Feng, "Reconfigurable 2-bit fixedfrequency beam steering array based on microstrip line," IEEE Trans. Antennas Propag., vol. 66, no. 2, pp. 683-691, February 2018. 